\title{
Light Transport through the Band-Edge States of Fibonacci Quasicrystals
}

\author{
Luca Dal Negro, ${ }^{1, *}$ Claudio J. Oton, ${ }^{1}$ Zeno Gaburro, ${ }^{1}$ Lorenzo Pavesi, ${ }^{1}$ Patrick Johnson, ${ }^{2}$ Ad Lagendijk,${ }^{2,3}$ \\ Roberto Righini, ${ }^{4}$ Marcello Colocci, ${ }^{4}$ and Diederik S. Wiersma ${ }^{4, \dagger}$ \\ ${ }^{1}$ INFM and Department of Physics, University of Trento, I-38050, Povo, Trento, Italy \\ ${ }^{2}$ Van der Waals-Zeeman Institute, University of Amsterdam, 1018 XE Amsterdam, The Netherlands \\ ${ }^{3}$ Department of Applied Physics \& MESA + Research Institute, University of Twente, Enschede, The Netherlands \\ ${ }^{4}$ INFM and European Laboratory for Non-Linear Spectroscopy, 50019 Sesto-Fiorentino (Florence), Italy
}

(Received 4 July 2002; published 3 February 2003)

\begin{abstract}
The propagation of light in nonperiodic quasicrystals is studied by ultrashort pulse interferometry. Samples consist of multilayer dielectric structures of the Fibonacci type and are realized from porous silicon. We observe mode beating and strong pulse stretching in the light transport through these systems, and a strongly suppressed group velocity for frequencies close to a Fibonacci band gap. A theoretical description based on transfer matrix theory allows us to interpret the results in terms of Fibonacci band-edge resonances.
\end{abstract}

DOI: $10.1103 /$ PhysRevLett.90.055501

The propagation of optical waves in complex dielectric systems is an intriguing research subject. Complex dielectrics are dielectric structures in which the refractive index varies over length scales comparable to the wavelength of light. In disordered materials light waves undergo a multiple scattering process and are subject to unexpected interference effects [1]. One of the first phenomena studied in this context was coherent backscattering [2] or weak localization of light [3]. Multiple light scattering in disordered dielectrics shows many similarities with the propagation of electrons in semiconductors, and various phenomena that are known for electron transport also appear to have their counterpart in optics. Important examples are the optical Hall effect and optical magnetoresistance [4], universal conductance fluctuations of light waves [5], optical negative temperature coefficient resistance [6], and Anderson localization of light [7]. In the case of Anderson localization, the propagation of light is even completely dominated by interference effects and the effective transport comes to a halt.

On the other extreme, periodic dielectric structures behave as a crystal for light waves. In periodic structures the interference is constructive in well-defined propagation directions, which leads to Bragg scattering and refraction. At high enough refractive index contrast, propagation is prohibited in any direction within a characteristic range of frequencies. This phenomenon is referred to as a photonic band gap [8] in analogy with the electronic band gap in a semiconductor. Whereas the knowledge on the propagation of light waves in completely ordered and disordered structures is now rapidly improving, little is known about the behavior of optical waves in the huge intermediate regime between total order and disorder.

Quasicrystals are nonperiodic structures that are constructed following a simple deterministic generation rule [9]. If made from dielectric material, the resulting struc-
PACS numbers: $61.44 . \mathrm{Br}, 42.25 . \mathrm{Bs}, 42.25 . \mathrm{Dd}, 42.25 . \mathrm{Hz}$

ture has fascinating optical properties. Quasicrystals of the Fibonacci type, for instance, exhibit an energy spectrum that consists of a self-similar Cantor set with zero Lebesgue measure [10]. The transmission spectrum of a Fibonacci system also contains forbidden frequency regions called "pseudo band gaps" similar to the band gaps of a photonic crystal [11]. In the frequency regime outside these Fibonacci band gaps, the light waves are critically localized. In contrast with the fully disordered (Anderson) localized case, these critically localized states decay weaker than exponentially, most likely by a power law, and have a rich self-similar structure [12]. This makes these systems very interesting for light localization studies, as proposed by Kohmoto et al. [13]. The first Fibonacci sequence for electron transport studies was realized by Merlin et al. [14], which was followed by several experiments and theoretical studies on electron propagation in these systems [15]. The experimental work on light transport in this fascinating class of structures is limited so far. Important pioneering experiments were performed by Gellermann et al. [16] who observed selfsimilarity in the transmission spectrum of Fibonacci dielectric multilayers and by Hattori et al.[17] who measured the Fibonacci dispersion curves.

A Fibonacci quasicrystal is a deterministic aperiodic structure that is formed by stacking two different compounds $A$ and $B$ according to the Fibonacci generation scheme: $S_{j+1}=\left\{S_{j-1} S_{j}\right\}$ for $j \geq 1$, with $S_{0}=\{B\}$ and $S_{1}=\{A\}$. The lower order sequences are $S_{2}=\{B A\}, S_{3}=$ $\{A B A\}, S_{4}=\{B A A B A\}$, etc. We limit ourselves in this Letter to one-dimensional structures. The advantage of 1D structures is that their optical properties can be calculated accurately and that samples can be designed and realized in any desired configuration. Since one has control over both layer thickness and refractive index, in principle any structure from completely ordered to completely disordered can be realized and studied experimentally. 
The quasicrystals for our experiments were realized using porous silicon multilayers. Fibonacci quasicrystals of up to 233 layers (Fibonacci order $j=12$ ) were obtained using a well-characterized electrochemical dissolution procedure [18]. The $p^{+}$-type doped $(0.01 \Omega \cdot \mathrm{cm})$ silicon substrates were etched in a $2: 1$ solution of ethanol and aqueous $48 \mathrm{wt} \%$ HF. Two distinct building blocks ( $A$ and $B$ ) were stacked according to the Fibonacci generation rule. Layer $A$ corresponds to a $157 \mathrm{~nm}$ thick layer with $69 \%$ porosity and refractive index 1.6 . Layer $B$ corresponds to a $105 \mathrm{~nm}$ thick layer with $47 \%$ porosity and refractive index 2.2. The total thickness of the 233 layer sample was $29 \mu \mathrm{m}$. The chemical etching process introduces a well-known thickness and porosity gradient, which in our case is about $6 \%$, respectively, $10 \%$, over the whole sample thickness [18]. (The layer thickness decreases and the porosity increases with depth.) We find that the Fibonacci band gap and most band edge states are robust against this small drift.

To study the transport properties of the Fibonacci band edge modes we have performed time-resolved transmission experiments using a fixed Mach-Zehnder interferometer coupled with a Michelson interferometer to measure the interferometric cross correlation of the transmitted pulse with a reference pulse. This technique provides both the amplitude and phase information of the transmission through the sample. For further details, we refer to Ref. [19]. As a laser source we used a tunable parametric oscillator, pumped by a fast (200 fs pulse duration) Ti:sapphire laser.

In Fig. 1 the experimental transmission spectrum of a 12th-order Fibonacci sample is shown. The inset shows three examples of the power spectrum of the incident laser pulses close to the low-energy band edge of

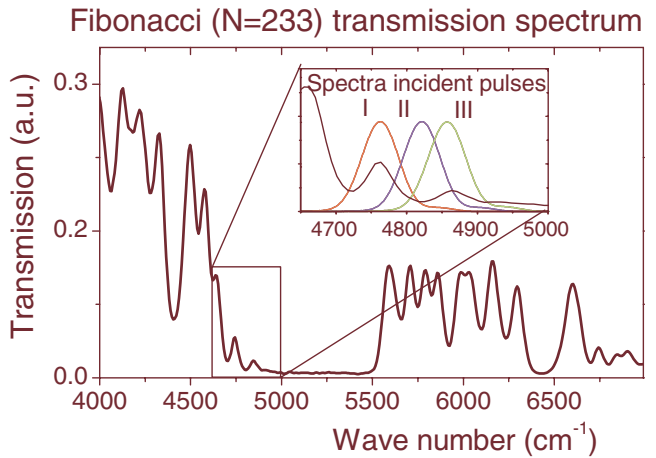

FIG. 1 (color online). Measured transmission spectrum of a 12th order Fibonacci $(N=233)$ quasicrystal. Around wave numbers $5000-5500 \mathrm{~cm}^{-1}$ the system exhibits a Fibonacci pseudo gap. The inset shows three examples of the power spectrum of the incoming laser pulses in the time-resolved experiment reported in Fig. 2. The spectrum was recorded with a standard Perkin and Elmer IR absorption spectrometer, and hence the transmission peaks are broadened due to the angular spread of the incoming light on the sample and lateral sample inhomogeneities. the Fibonacci pseudogap around wave numbers $5000-5500 \mathrm{~cm}^{-1}$. The measured pulse envelopes of the time-resolved transmittance for these three examples are plotted in Fig. 2. When the incoming pulse is resonant with one transmission peak, the pulse is significantly delayed and stretched. This stretching becomes surprisingly strong close to the band edge (e.g., Fig. 2, curve IIIa). In addition to the delay and stretching, when the spectrum of the laser pulse overlaps with two adjacent narrow transmission modes a strongly oscillatory behavior is observed (e.g., Fig. 2 curve IIa). These oscillations can be interpreted as due to beating between individual band-edge modes. Indeed the frequency of the oscillations corresponds to the frequency difference between the peaks in the transmission spectrum.

The time delay $\Delta t$ and the decay constant $\tau$ of the transmitted pulse are plotted in Fig. 3. The time delay was calculated from the delay of the center of mass of the transmitted pulse envelopes relative to the undisturbed pulse (a pulse that has passed through the underlying substrate but not through the Fibonacci system). The decay constant $\tau$ was obtained by an exponential fit to the pulse decay envelope at long times. Both $\Delta t$ and $\tau$ elongate strongly for pulse energies close to the band edge. The strong pulse delay leads to a group velocity suppression, as can be seen in Fig. 3(b). The group velocity reduction obtained in these structures is 3 times larger than that observed in three-dimensional photonic crystals made of colloidal polystyrene spheres [20].

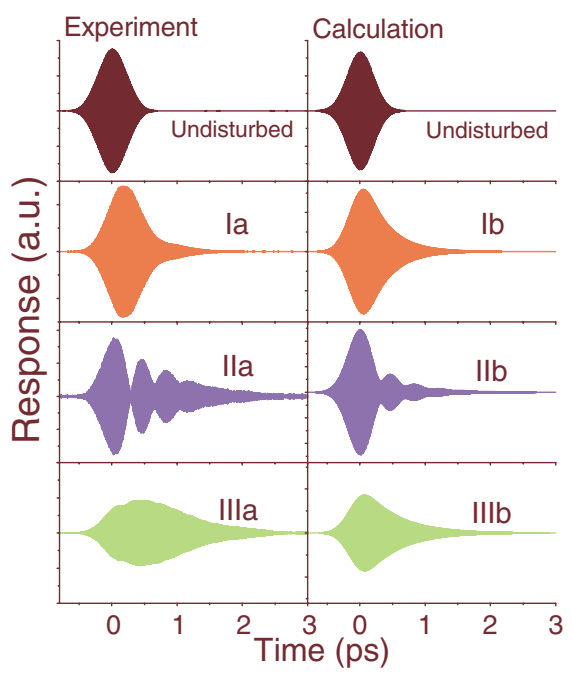

FIG. 2 (color online). Experimental data and calculation of the transmission through Fibonacci samples at four different frequencies. Also the undisturbed pulse, which has passed through only the Si substrate and not the Fibonacci sample, is plotted for comparison. The time offset corresponding to the total optical thickness of the sample has been subtracted in all cases. When the laser pulse is resonant with one band edge state the transmitted intensity is strongly delayed and stretched. When two band edge states are excited, mode beating is observed. 


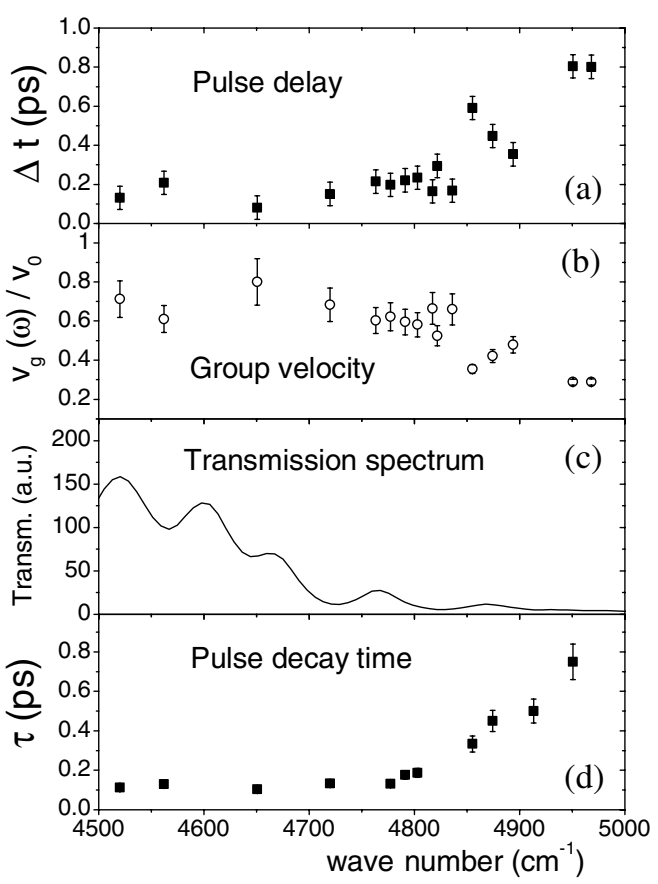

FIG. 3. (a) Time delay of the center of mass of the transmitted pulse with respect to the undisturbed pulse. (b) Group velocity with respect to the vacuum group velocity, as derived from the time delay. (c) Detail of the experimental transmission spectrum of the 12th order Fibonacci sample. (d) Decay time of the exponentially decaying transmitted pulse at long times.

The large group velocity reduction and pulse stretching that we find experimentally is observed only close to the band edge of the Fibonacci spectrum. The band edge is also the region where the periodic-like features (band gap) of the Fibonacci system go over into its disorder properties (critically localized states). Inspired by our experimental results we decided to look in more detail into the physical nature of these Fibonacci band edge states.

We calculated the field distribution of the experimentally studied modes in our Fibonacci quasicrystal, following a standard scattering-states method [21]. The magnitude of the electric $(E)$ and magnetic $(B)$ fields at the left interface of a dielectric layer is simply related to $E$ and $B$ at the right interface of the same layer through the matrix relation [22]:

$$
\left(\begin{array}{c}
E_{\ell} \\
B_{\ell}
\end{array}\right)=M\left(\begin{array}{c}
E_{r} \\
B_{r}
\end{array}\right)=\left(\begin{array}{cc}
\cos \delta & i \frac{\sin \delta}{\gamma} \\
i \gamma \sin \delta & \cos \delta
\end{array}\right)\left(\begin{array}{c}
E_{r} \\
B_{r}
\end{array}\right)
$$

where the indices $\ell$ and $r$ refer to the left and right interfaces of the layer, $\delta=k_{0} n d$ is the phase change, and $\gamma$ is the inverse of the light velocity across the layer ( $k_{0}=$ wave vector in vacuum, $n=$ refractive index, $d=$ layer thickness). From the continuity requirements of the electric and magnetic fields, one can construct the transfer matrix $M$ of the whole system by simple matrix multi- plication. For the layer thickness $d$ and refractive index $n$ we take exactly the values of our samples as given before. The response in the time domain can then be calculated from the inverse Fourier transformation of the product of the complex transmission coefficient and the incident pulse envelope. Taking exactly the experimental incidence pulse envelope (FWHM: $63 \mathrm{~cm}^{-1}$ ), coherent beating and pulse stretching are very well reproduced in the calculation, as can be seen in Fig. 2 .

For one-dimensional structures, it is also possible to compute the electric field distribution inside the sample. The reflectivity coefficient of the whole sample must be calculated previously, after which the incident and the total reflected light can be used as boundary conditions to compute the field amplitude of all layers inside the structure. The electric field on the $k$ th interface can be expressed as

$$
E\left(z^{(k)}, \omega\right)=\{1+r(\omega)\} m_{22}^{(k)}-\gamma_{0}\{1-r(\omega)\} m_{12}^{(k)},
$$

where $m_{\alpha \beta}^{(k)}$ are the elements of the transmission matrix from the first to the $k$ th interface. The intensity is then simply the square of the electric field, as plotted in Fig. 4. The Fibonacci pseudo band gap is clearly visible in the lower part of the plot. Just above the pseudo band gap the band edge modes are visible which correspond to the transmission peaks in the inset of Fig. 1. The insets show the normalized field intensity distributions for
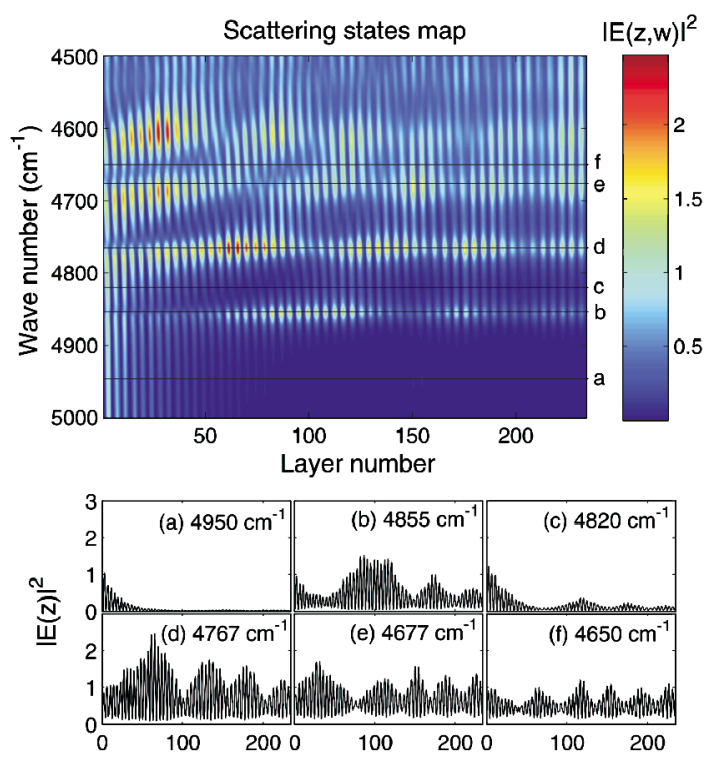

FIG. 4 (color). Calculated intensity distribution inside the Fibonacci quasicrystal sample as used in the experiments. $x$ axis: layer number; $y$ axis: wave number of the incident light. This means that any horizontal cut through the graph represents an intensity distribution at one specific frequency. The insets show the intensity distributions at several frequencies. (Horizontal cuts are indicated by black lines in the color graph.) The input intensity of the electric field has been normalized to unity. Note that the intensity inside the sample can become larger than 1 , due to internal resonances. 
several frequencies. The incoming field is normalized to 1. The first inset (a) shows the exponentially decaying intensity of light that is incident in the band gap region, whereas the other insets (b)-(f) show the intensity destribution in the band edge region when moving away from the band gap. [Insets (b) and (d) correspond to the first two transmission maxima, whereas (c) is taken in between these maxima.] The first and second order band edge resonances (with only one, respectively, two maxima) are suppressed due to the minor drift in layer thickness and porosity.

The resonances of the band edge states are sharp enough to allow for the occurrence of mode beating when adjacent modes are excited simultaneously, as observed in the experiments. Figure 4 also illustrates nicely the field-enhancement effect: close to the band edge the field inside the sample becomes locally larger than 1 . The distributions that we find in Fig. 4 have a notable similarity to the band edge resonances occurring in photonic crystals [23] but are less regular. Band edge resonances in (finite-size) photonic crystals are due to a transient standing wave that is formed inside the sample and can temporarily store a substantial amount of energy. This is consistent with a large group velocity reduction and strong pulse stretching as observed in our experiments. Since this transient standing wave is formed from reflection by the sample bounderies, it has the characteristic intensity distribution of the various harmonics of a standing wave. Band edge resonances in photonic crystals are not localized states since their extension scales linearly with the system size and they do not decay to zero [23]. In contrast, the Fibonacci band edge resonances will decay via a power law due to their critically localized nature.

Summarizing, we find that the Fibonacci band edge states exhibit mode beating, a sizable field enhancement, and a group velocity reduction that is 3 times larger than that observed in three-dimensional colloidal photonic crystals of polystyrene. As in the photonic crystal case the Fibonacci band edge states exhibit band edge resonances which are, however, critically localized due to the Fibonacci disorder. Fibonacci systems can provide an interesting alternative to regular photonic crystals for the realization of photonic devices, such as optical filters with a self-similar spectrum and a high wavelength selectivity in the band edge region. Another interesting future application of these materials could be realized in the field of random lasers, where the Fibonacci band edge resonances could serve as a new type of complex cavity that provides the feedback for laser action.

We acknowledge inspiring discussions with Professor Zhao-Qing Zhang and Professor V. Freilikher. This work has been financially supported by the MARC project of University of Trento, by the RANDS project of section E of the INFM, and by the EC under Contract No. HPRICT1999-00111.
*Electronic address: dalnegro@ science.unitn.it

†Electronic address: wiersma@lens.unifi.it

[1] See, for instance, Ping Sheng, Introduction to Wave Scattering, Localization, and Mesoscopic Phenomena (Academic Press, New York, 1995).

[2] Y. Kuga and A. Ishimaru, J. Opt. Soc. Am. A 1, 831 (1984).

[3] M. P. van Albada and A. Lagendijk, Phys. Rev. Lett. 55, 2692 (1985); P. E. Wolf and G. Maret, Phys. Rev. Lett. 55, 2696 (1985).

[4] B. A. van Tiggelen, Phys. Rev. Lett. 75, 422 (1995); A. Sparenberg, G. L. J. A. Rikken, and B. A. van Tiggelen, Phys. Rev. Lett. 79, 757 (1997).

[5] F. Scheffold and G. Maret, Phys. Rev. Lett. 81, 5800 (1998).

[6] D. S. Wiersma, M. Colocci, R. Righini, and F. Aliev, Phys. Rev. B 64, 144208 (2001).

[7] S. John, Phys. Rev. Lett. 53, 2169 (1984); P.W. Anderson, Philos. Mag. 52, 505 (1985).

[8] E. Yablonovitch, Phys. Rev. Lett. 58, 2059 (1987); S. John, Phys. Rev. Lett. 58, 2486 (1987).

[9] T. Fujiwara and T. Ogawa, Quasicrystals (SpringerVerlag, Berlin, 1990).

[10] Godfrey Gumbs and M. K. Ali, Phys. Rev. Lett. 60, 1081 (1988).

[11] See, e.g., Franco Nori and J. P. Rodriguez, Phys. Rev. B 34, 2207 (1986); R. B. Capaz, B. Koiller, and S. L. A. de Queiroz, Phys. Rev. B 42, 6402 (1990).

[12] T. Fujiwara, M. Kohmoto, and T. Tokihiro, Phys. Rev. B 40, 7413 (1989); C. M. Soukoulis and E. N. Economou, Phys. Rev. Lett. 48, 1043 (1982).

[13] Mahito Kohmoto, Bill Sutherland, and K. Iguchi, Phys. Rev. Lett. 58, 2436 (1987); B. Sutherland and M. Kohmoto, Phys. Rev. B 36, 5877 (1987).

[14] R. Merlin, K. Bajema, R. Clarke, F.Y. Juang, and P. K. Bhattacharya, Phys. Rev. Lett. 55, 1768 (1985).

[15] J. B. Sokoloff, Phys. Rev. Lett. 58, 2267 (1987); Ch. Wang and R. A. Barrio, Phys. Rev. Lett. 61, 191 (1988); E. Maciá and F. Domínguez-Adame, Phys. Rev. Lett. 76, 2957 (1996); F. Piéchon, Phys. Rev. Lett. 76, 4372 (1996); X. Huang and Ch. Gong, Phys. Rev. B 58, 739 (1998); F. Steinbach, A. Ossipov, Tsampikos Kottos, and T. Geisel, Phys. Rev. Lett. 85, 4426 (2000).

[16] W. Gellermann, M. Kohmoto, B. Sutherland, and P.C. Taylor, Phys. Rev. Lett. 72, 633 (1994).

[17] T. Hattori, N. Tsurumachi, S. Kawato, and H. Nakatsuka, Phys. Rev. B 50, 4220 (1994).

[18] A. G. Cullis, L. T. Canham, and P. D. J. Calcott, J. Appl. Phys. 82, 909 (1997); L. Pavesi, Riv. Nuovo Cimento 20, 10 (1997).

[19] R. H. J. Kop and R. Sprik, Rev. Sci. Instrum. 66, 5459 (1995).

[20] A. Imhof, W. L. Vos, R. Sprik, and A. Lagendijk, Phys. Rev. Lett. 83, 2942 (1999).

[21] A. Kavokin, G. Malpuech, A. Di Carlo, P. Lugli, and F. Rossi, Phys. Rev. B 61, 4413 (2000).

[22] F. L. Pedrotti and L.S. Pedrotti, Introduction to Optics (Prentice-Hall, Englewood Cliffs, NJ, 1987).

[23] M. Scalora et al., Phys. Rev. E 54, R1078 (1996). 